Article

\title{
Theoretical Study: High Harmonic Generation by Light Transients
}

\author{
Maximilian Wendl ${ }^{1}$, Maximilian Högner ${ }^{1}$ (i) and Hanieh Fattahi ${ }^{1,2, *(D)}$ \\ 1 Department für Physik, Ludwig-Maximilians-Universität München, Am Coulombwall 1, \\ D-85748 Garching, Germany; max.wendl@physik.uni-muenchen.de (M.W.); \\ Maximilian.Hoegner@physik.uni-muenchen.de (M.H.) \\ 2 Max-Planck Institut für Quantenoptik, Hans-Kopfermann-Str. 1, D-85748 Garching, Germany \\ * Correspondence: hanieh.fattahi@mpq.mpg.de; Tel.: +49-89-3290-5732
}

Received: 11 April 2018; Accepted: 2 May 2018; Published: 5 May 2018

\begin{abstract}
The dynamic of electron densities in matter upon the interaction with an intense, few-cycle electric field of light causes variety of nonlinear phenomena. Capturing the spatiotemporal dynamics of electrons calls for isolated attosecond pulses in the X-ray regime, with sufficient flux to allow for: (i) attosecond pump-attosecond probe spectroscopy; or (ii) four-dimensional imaging. Light field synthesizers generate arbitrary sub-cycle, non-sinusoidal waveforms. They have a great potential to overcome the limitations of current laser sources and to extend attosecond pulses towards the X-ray regime. In this paper, we show theoretically how the achievable high-energy, high-power waveforms from current light field synthesizers can be optimized to enhance the harmonic yield at high photon energies and can serve as a promising source for scaling the photon energies of attosecond pulses. We demonstrate that the simulated optimized, non-sinusoidal waveform in this work can increase the photon flux of $\mathrm{keV}$, attosecond pulses by five orders of magnitude compared to the achievable flux from longer wavelength sources and at similar photon energies.
\end{abstract}

Keywords: ultrafast nonlinear optics; X-rays; soft X-rays; extreme ultraviolet; ultrafast lasers; ultrafast spectroscopy

\section{Introduction}

On the very fundamental level, electron density oscillations in an atomic-scale environment induced by intense light are the origin of many nonlinear phenomena. This induced electron density can be steered and controlled which holds promise for dielectric optical switching at clock rates [1]. Controlling the electron density in solids benefit from the availability of a shorter driving field down to sub-cycle duration. It is also of great interest to simultaneously study the spatiotemporal dynamics of electrons. Therefore, these current studies benefit from the availability of sources which: (i) generate sub-cycle pulses; and (ii) allow for simultaneous four-dimensional imaging of electron dynamics at its ultimate resolution.

During the past decade, pump-probe attosecond spectroscopy, by means of synchronized few-cycle pump pulses with extreme-ultraviolet (XUV), attosecond probe pulses, has enabled the direct measurement of many fundamental processes [2]. However, resolving electron dynamics in solids in their ultimate spatiotemporal resolution calls for extending the photon energy of isolated attosecond pulses to $\mathrm{X}$-rays, with sufficient photon flux for imaging.

Non-sinusoidal, sub-cycle waveforms can be generated by field synthesis of several few-cycle pulses [3] and when the same source is used for generation of attosecond, X-ray pulses with sufficient flux, sub-cycle nonlinearities and electron dynamics can be explored. 
In high harmonic generation (HHG), an increase in the period of the oscillating laser field leads to the increase in the electron's excursion time and therefore recollision energy and HHG cutoff. The longer wavelength of the driving field results in a higher excursion time $\tau_{e x} \propto \lambda_{0}$ of the freed electrons. However, quantum diffusion scales with $\lambda^{-5}$ to $\lambda^{-7}$, which dramatically reduces the recombination probability with the parent ion $\left(\propto \tau^{-3}\right)$ and thus the yield of the harmonic radiation at $\mathrm{keV}$ photon energies [4].

On the other hand, the HHG cutoff scales linearly with the laser's peak intensity. However, increasing the peak intensity of pulses containing more than one optical cycle leads to undesired pre-ionization of the gas atoms, causing depletion of their ground state, reducing the phase-matching during pulse propagation and ultimately leading to an exponential drop in harmonic yield $[5,6]$.

High peak intensity also causes spatiotemporal distortions within the driving pulse. Defocusing and self-phase modulation reduces the peak intensity as the pulse propagates through the gas, thereby reducing the yield of high energy photons [7,8]. In addition, the HHG yield depends on the coherent build-up of radiation emitted from many atoms, which is optimal when both the driving pulse and the generated light travel in phase through the medium. This condition is achieved when the positive dispersion of the neutral gas is balanced by the negative dispersion of the free electrons. However, this is only possible below a critical ionization rate, therefore favouring long wavelength driving fields, as they require a lower intensity to generate a specific cutoff energy. Generation of excessive free electrons in the medium at high peak intensity results in a phase mismatch [9].

The quantum phase of the electron wavefunction returning to the core can be optimized by shaping the laser field to enhance a particular harmonic feature or the intra-atomic phase matching. Therefore, the yield and efficiency of the HHG process can be increased by synthesis of two or three color sinusoidal pulses [10-13] due to the enhanced tunnel ionization [14,15]. However, this approach suffers from pre-ionization and ground state depletion, due to the multiple field cycles in the interfering pulses and their incomplete destructive field interference over tens of femtoseconds out of the temporal window of interest for HHG. Non-sinusoidal waveforms can be generated by field synthesis of few-cycle pulses at different central frequencies to confine the field's energy and therefore ionization within a single cycle electric field.

To overcome these barriers and to push HHG cutoff energies beyond the keV levels, all the above-mentioned parameters must be carefully tailored and optimized. Despite preliminary studies on the waveform synthesis of few-cycle pulses $[6,13,16]$, there has not been systematic investigation on optimized drivers for generating isolated attosecond pulses in the $\mathrm{X}$-ray regime.

In this work, we address how temporal field synthesis of a few-cycle pulse at $2 \mu \mathrm{m}$ with weaker few-cycle pulses at the second and third harmonic of its carrier frequency leads to a non-sinusoidal temporal shape which fulfills the above-mentioned criteria. Field synthesis of few-cycle pulses allows for tailoring of the light's electric field at sub-cycle scale and consequently controlling electron trajectories and optimization of harmonic yields. In this study, our focus will be on generation of isolated attosecond pulses. Even though we limited ourselves to the dipole response of a single atom, the ionization rate caused by each waveform is also optimized, as it is of great importance when considering propagation and phase-matching in the gas.

Non-sinusoidal waveforms, scalable in average-power and energy, can be generated from waveform synthesizers based on Yb:YAG laser technology [17-19]. Here, a high-energy, high-power Yb:YAG laser serves as the pump source [20]. A multi-octave, low-energy seed spectrum with a carrier-envelope phase stability is generated directly from the Yb:YAG amplifier [21] and divided to several spectral regions by using a broadband beam splitter [22]. Each spectral region is amplified to higher energies in multi-stage optical parametric amplifiers pumped by different harmonics of the Yb:YAG amplifier [23]. Eventually, the broadband amplified pulses are compressed into few-cycle pulses, and coherently recombined (see Figure 1). This laser structure serves as a promising tool for extending the HHG cutoff to $\mathrm{keV}$ photon energies, as here: (i) the addition of the longer wavelengths to the driving field enables the recollision of accelerated electrons with more energy; (ii) confining the 
multioctave field down to a single field cycle at multi-terawatt peak power level enables full control over ionization [24]; and (iii) tailoring the chirp of the waveform allows for better control of tunneling ionization, releasing the electrons and of their subsequent sub-cycle trajectories.

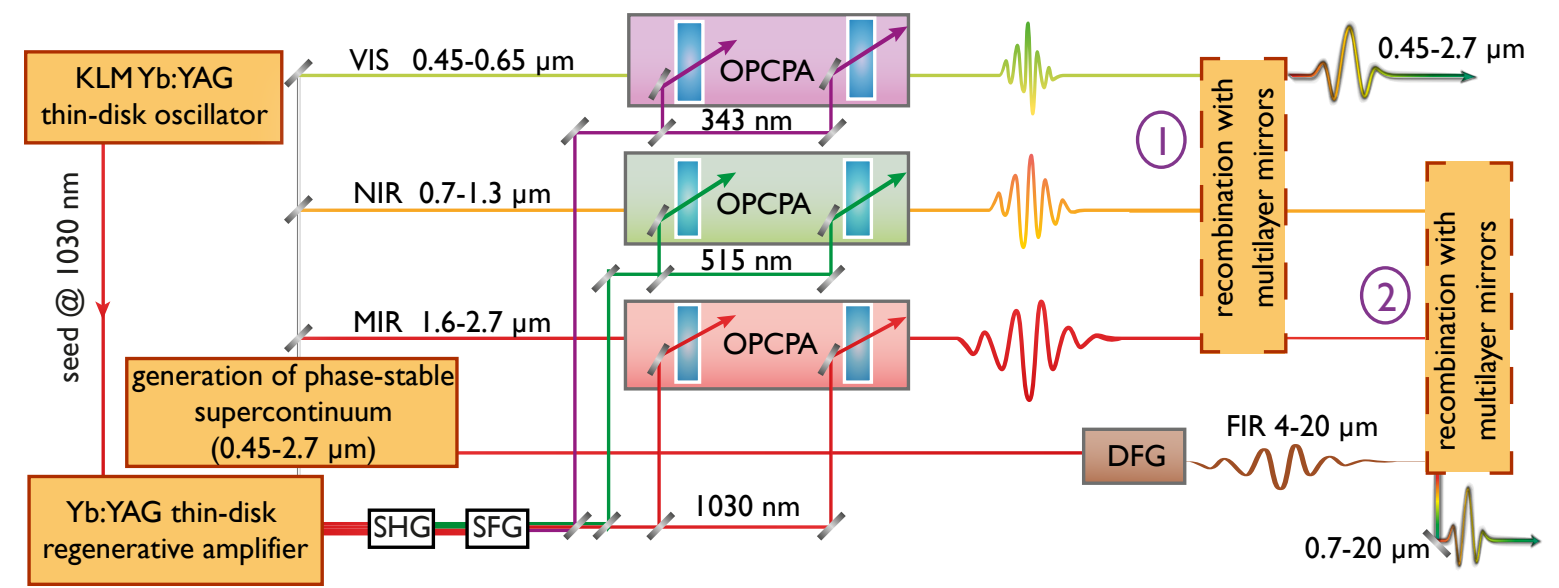

Figure 1. Schematic architecture of a three-channel optical parametric chirped pulse amplifier (OPCPA) field synthesizer seeded and pumped by picosecond Yb:YAG lasers. A part of the output of the Yb:YAG amplifier is used for generating a phase-stable multi-octave super-continuum seed, which is then split into three channels, centered at $550 \mathrm{~nm}, 1 \mu \mathrm{m}$ and $2 \mu \mathrm{m}$, respectively. The different channels are pumped by different harmonics of the multi-mJ level kHz, Yb:YAG regenerative amplifier output. Each channel supports few-cycle pulses. By coherently combining the three few-cycle channels, light transients spanning from 0.45 to $2.7 \mu \mathrm{m}$ can be generated (1). The sub-cycle waveforms can be furthermore extended to far-infrared by difference frequency generation (DFG) of the seed pulses (2). The figure is adopted from [17]. FIR: far-infrared; MIR: mid-infrared; NIR: near-infrared; VIS: visible; SHG: second harmonic generation; SFG: sum frequency generation.

Figure 2 compares the single dipole response of a $5 \mathrm{fs}$, Gaussian pulse at $800 \mathrm{~nm}$ achievable from the current Ti:Sa laser technology with the dipole response of the sub-cycle waveform generated from a similar laser structure discussed in Fattahi et al. [18]. The spectra used for generation of such a waveform are shown in Figure 2a, with broadband spectra centered at $540 \mathrm{~nm}$ (visible (VIS), $880 \mathrm{~nm}$ (near-infrared (NIR), and $2.1 \mu \mathrm{m}$ (mid-infrared(MIR). The energy distribution between the three spectra for the shown synthesized waveform in Figure $2 b$ is (VIS:NIR:MIR) 0.3:0.85:1, which is the dictated ratio for having the maximum optical-to-optical conversion efficiency from the described laser structure. The three spectra with zero spectral phase, are coherently combined at zero delay and the carrier-envelope phase (CEP) of the resulting waveform is scanned for generation of the highest cutoff energy. For this simulation, we used a nonadiabatic model based on the modified Lewenstein model [25-27].

At the similar peak intensity of $52.8 \times 10^{14} \mathrm{~W} / \mathrm{cm}^{2}$ and when both waveforms have interacted with $\mathrm{He}$, the HHG spectrum of the sub-cycle waveform reaches $1.7 \mathrm{keV}$, generating a 1.7 times higher cutoff than the Gaussian pulses at $800 \mathrm{~nm}$ (Figure 2c).

As it is shown in the spectrogram of the light-transient in Figure 2c, the substantial ionization is limited to one optical half-cycle, obviating the need for spectral filtering or sophisticated gating techniques which are limiting the obtainable bandwidth. However, in this case, a fraction of about $70 \%$ of the atom is ionized. The presented calculation shows that although sub-cycle pulses are required and interesting for many pump-probe experiments, they are not the optimal approach for generating $\mathrm{keV}$ photons. 

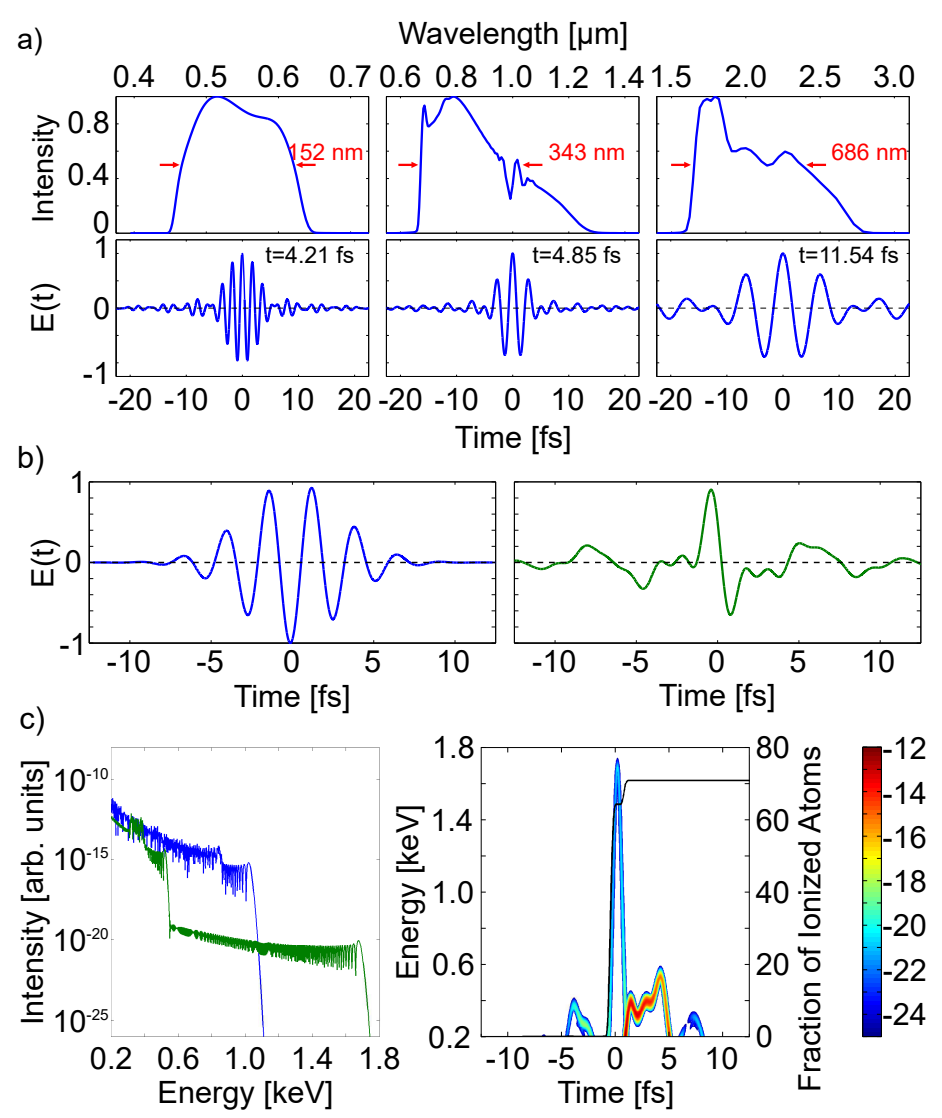

Figure 2. (a) Calculated spectral intensity of pulses achievable from an OPCPA based field synthesizer (top) and their corresponding Fourier transform limited electric field (bottom). (b) The electric field of a Gaussian, $5 \mathrm{fs}$ pulses at $800 \mathrm{~nm}$ (left) and the synthesized pulse from the spectra shown in (a) with an energy ratio of 0.3:0.85:1. (c) Overlaid HHG spectra of the $5 \mathrm{fs}$ pulses at the central wavelength of $800 \mathrm{~nm}$ and $60^{\circ}$ carrier-envelope phase (CEP), and the synthesized pulses at the CEP of $67^{\circ}$, interacting with He (left). The spectrogram of the synthesized pulse and the induced rate of ionization (black) in He (right).

\section{Method}

Based on temporal or spectral synthesis, arbitrary non-sinusoidal waveforms can be generated by superimposing several pulses at different carrier frequencies and with different amplitude and phase. Therefore, the relative energy or temporal delay of several few-cycle pulses can be varied to generate different waveforms as shown in Figure 3.

\subsection{Dipole Response Calculation}

For finding the optimum waveform for $\mathrm{keV}$ photon generation, we calculated the single-dipole response of He gas in interaction with different waveforms. We chose $15 \times 10^{14} \mathrm{~W} / \mathrm{cm}^{2}$ peak intensity for the following simulations to keep the total ionization below the critical ionization level of helium [28].

The time axis for the dipole response calculation was defined in co-moving coordinates as

$$
t_{c m c}=2 \pi\left(-t_{\text {win }}: 1 / t_{\text {step }}: t_{\text {win }}\right),
$$

where $t_{\text {win }}=p_{x} c / \lambda$ was calculated from the time axis limits $p_{x}$ in fs that were to be resolved and $t_{\text {step }}$ determines the number of calculated harmonics. To reduce simulation time, both values should be chosen as low as possible. For this reason, $t_{\text {win }}=40 \mathrm{fs}$ and $t_{\text {win }}=75 \mathrm{fs}$ was used for pulses without 
and with delay, respectively, and $t_{\text {step }}$ was chosen such that a specific energy of the harmonic radiation could be resolved. The parameters for the time integration in the Lewenstein model were chosen such that contributions of both long and short trajectory electrons are included [25].

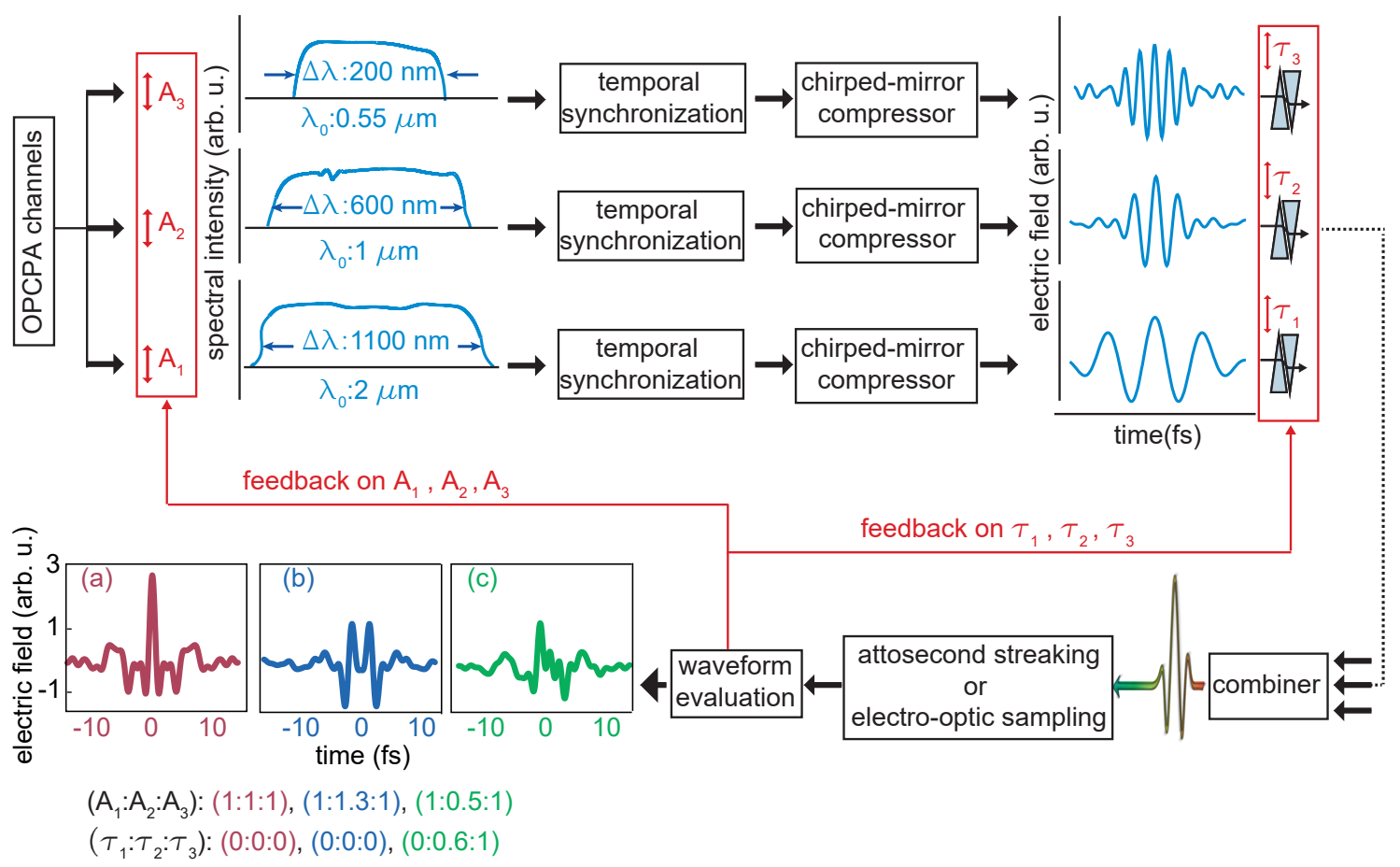

Figure 3. Detailed setup of the temporal synthesis of the VIS, NIR and MIR pulses. The amplified spectra of all three OPCPA channels centered at $0.55 \mu \mathrm{m}, 1 \mu \mathrm{m}$, and $2 \mu \mathrm{m}$ are first sent through a delay line to achieve a temporal overlap between the synthesizer's three arms. Thereafter, the temporally synchronized pulses are compressed to their Fourier transform limit in each arm separately using a set of broadband dielectric chirped mirrors. After passing through a pair of glass wedges to fine tune the relative delay between each arm the three compressed pulses are spatially combined in two broadband dielectric beam combiners. The generated light transients are evaluated using attosecond streaking [29] or electro-optic sampling [30]. By adjusting the relative spectral amplitude of each arm $\left(A_{1}, A_{2}, A_{3}\right)$ and their relative phase $\left(\tau_{1}, \tau_{2}, \tau_{3}\right)$, a variety of transients can be generated. As examples, Panels (a-c) show three differently synthesized transients. The figure is adopted from [17].

The cutoff energy was taken as the last point in the dipole response spectrum before the intensity drops below $10^{-23}$ and the harmonic yield was calculated as the maximum of the last peak in the high harmonic spectrum.

\subsection{Pulse Synthesis}

Three realistic spectra [18] centered in the VIS at $540 \mathrm{~nm}$, the NIR at $880 \mathrm{~nm}$ and the MIR at $2.1 \mu \mathrm{m}$ were used for the pulse synthesis. Before they were combined, each individual spectrum was normalized to its energy content and then multiplied by a factor between 0 and 1 to define the energy ratio between them. The delay between the pulses was set by adding a linear term in the spectral phase to the individual spectra. Here, it was ensured that they still overlapped, thus forming a single pulse. Afterwards, the spectra were combined and the overall CEP of the resulting waveform was altered by adding a zero order phase. The pulse in time-domain was calculated by the fast Fourier transform of the combined spectrum. To ensure a constant peak intensity of $15 \times 10^{14} \mathrm{~W} / \mathrm{cm}^{2}$, each waveform was normalized to the maximum of its electric field envelope. 


\subsection{Scanning the Amplitude and the Delay}

The influence of the energy ratio and the delay between the three channels on the resulting high harmonic radiation were first analyzed individually. To this end, the amplitude of each channel was scanned from 0.1 to 1 with a step size of 0.05 . For selected amplitude ratios, the delay between the three channels was then altered from $-8 \mathrm{fs}$ to $+8 \mathrm{fs}$ with a step size of $0.25 \mathrm{fs}$. Here, each delay setting was shifted in time to be centered around $t=0$ to avoid cutting the pulse with the specified time window (Equation (1)).

To find waveforms leading to a cutoff energy above $1 \mathrm{keV}$, both energy ratio and delay were scanned simultaneously. Based on our initial analysis, the MIR amplitude was varied from 0.6 to 1 with a step size of 0.2 , while both VIS and NIR were scanned from 0.1 to 0.4 with a step size of 0.1 . The delay was scanned from -6 to +6 fs with a step size of 0.3 fs. To reduce the calculation time, the scan was performed in two steps. First, the dipole response was only calculated up to an energy of $1 \mathrm{keV}$ to eliminate amplitude and delay combinations resulting in sub-keV cutoff energies. The second step was to calculate the full dipole response of the remaining waveforms up to an energy of $1.7 \mathrm{keV}$.

\subsection{Ionization Rate}

As the driving field's photon energies used in this study lie well below the ionization potential of helium, the rate of tunnel ionization can be calculated analytically in the static field approximation. Therefore, the empirical formula proposed by Tong and Lin [26] was used, which corresponds to a correction of the analytical Ammosov-Delone-Krainov (ADK) formula for tunnel ionization, extending it to higher intensities. This is also implemented in the HHG [25] framework and has been shown to work very well for long wavelength driving fields in Helium [26].

\subsection{Spectrogram}

The spectrogram of the harmonic radiation was calculated by scanning a Gaussian gate of the form

$$
g(t)=\exp \left[-\frac{1}{2}\left(\frac{t-t_{0}}{\tau_{g}}\right)^{2}\right]
$$

with a time constant $\tau_{g}=60$ as across the pulse. The central position of the gate $\left(t_{0}\right)$ was therefore moved within a time window of $25 \mathrm{fs}$ and a step size of 20 as for pulses without delay. For waveforms that included a delay, the time window was increased to $45 \mathrm{fs}$ with a step size of 50 as.

\subsection{Electron Trajectories}

The emitted photons from the recolliding electrons with long trajectories have a lower contribution in the coherent build up and formation of the macroscopic high harmonic spectrum, as long trajectories have a steeper intensity dependent phase [31]. Therefore, the dipole response was recalculated including only short trajectory electrons.

\section{Results and Discussion}

Figure 4a shows how the HHG cutoff, ionization fraction, and yield is affected when changing the relative amplitude between the three superimposed pulses. The energy ratio between the three pulses is varied from $10 \%$ to $100 \%$. It can be observed that, while the ionization fraction for different energy ratios changes by less than $7 \%$, the harmonic yield varies over more than eight orders of magnitude. This simulation loop identifies the amplitude ratio of (VIS:NIR:MIR) 0.1:0.1:1 as the optimum ratio to generate the highest cutoff energy. The inset in Figure 4 shows the waveform generating the highest cutoff. The dominant role of the MIR in extending the cutoff was expected, due to the higher electron excursion time. 
a)

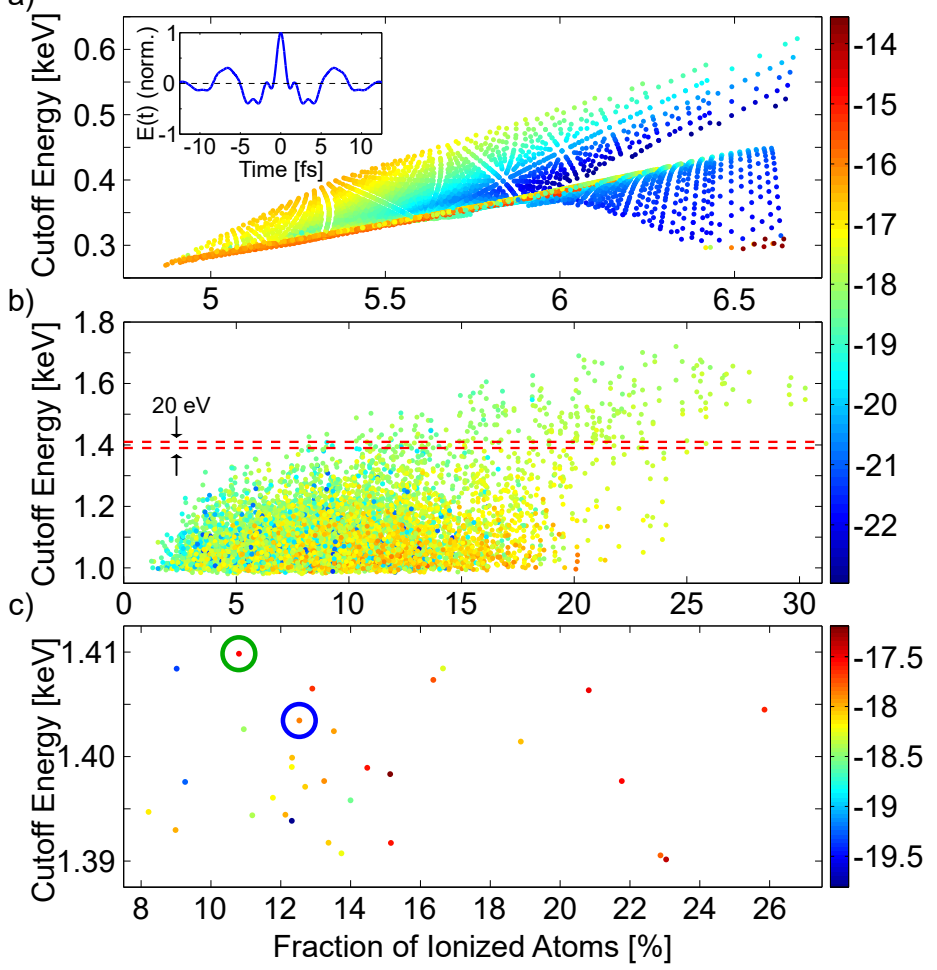

Figure 4. Relation between cutoff energy, ionization rate and harmonic yield for different: (a) energy ratio of the superimposed few-cycle pulses at zero delay; and (b) energies and delays. The highest cutoff energy (marked in Panel (a) with a red circle) corresponds to a waveform with energy ratio of 0.1:0.1:1. Inset shows the temporal electric field of the best waveform at zero delay. (c) The optimized and experimentally interesting waveforms in this energy region are marked in green and blue, respectively. The logarithmic color scale represents the harmonic yield in all three panels.

Figure $4 \mathrm{~b}$ shows how the ionization fraction, cutoff, and yield varies when scanning the relative amplitude and delay of the superimposed pulses simultaneously. It can be seen that, unlike the results shown in Figure 4a, the ionization rate varies dramatically for different inter-pulse delays. In Figure 4c, the yield and energy of different waveforms for a small energy interval of $20 \mathrm{eV}$ at $1.4 \mathrm{keV}$ is shown. Our model enabled us to identify the optimum waveform in terms of the harmonic yield and ionization rate which is marked with a green circle. The waveform has an amplitude ratio of 0.1:0.1:1 and the relative delay of $-6: 3.6:-0.3$ (fs). In addition, the other waveform marked with a blue circle is of particular interest, since it contains a relatively high amount of NIR pulses. This is an important factor as: (i) the quantum diffusion for such a waveform is smaller; and (ii) optical parametric amplifiers and optical components work more efficiently in this spectral region.

Figure $5 \mathrm{a}, \mathrm{b}$ illustrates the electric fields, ionization rates, and corresponding spectrograms of the optimized (green circle) and experimentally interesting (blue circle) waveforms. Both waveforms follow a similar trend: having a less than one-cycle MIR pulse, modulated on either the leading or trailing edge, or both edges by the fast oscillating fields in the NIR and VIS. This leads to a suppression of the electric field, except at the sub-cycle part of the electric field, which has a major role in generating the highest harmonics. In addition, the ionization rate prior to the arrival of the main sub-cycle of the electric field, and the launch as well as recollision of the high-energy trajectories, are controlled.

As shown in Figure $4 \mathrm{~b}$, at a relative inter-pulse delay of $-6:-1.5: 5.5(\mathrm{fs})$, the maximum photon energy of over $1.7 \mathrm{keV}$, with an ionization rate of $25 \%$ is reached. Compared to the light transient shown in Figure 2, this waveform leads to an order of magnitude higher harmonic yield and three time less ionization rate. 
a)

b)

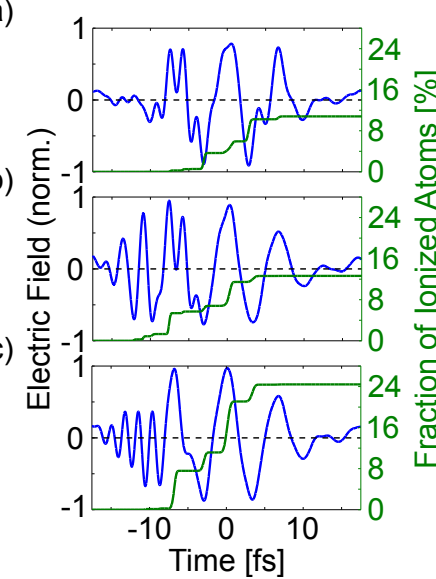

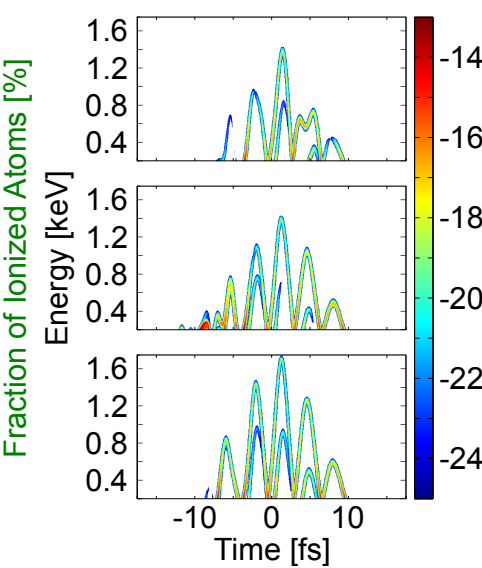

Figure 5. Electric field, ionization rate (left) and the spectrogram (right) of optimized waveforms with: (a) 0.1:0.1:1 energy ratio at -6:3.6:-0.3 (fs) delay generating $1.4 \mathrm{keV}$; (b) 0.1:0.3:1 energy ratio at $-2.7:-6: 4.8$ (fs) delay generating $1.4 \mathrm{keV}$; and (c) 0.1:0.1:1 energy ratio at $-6:-1.5: 5.4$ (fs) delay generating $1.7 \mathrm{keV}$. The logarithmic color scale in both panels represents the harmonic yield.

Table 1 compares the ionization rate, required peak intensity, and the harmonic yield for generating $1.7 \mathrm{keV}$ isolated attosecond pulse, for six different cases. The driving field in case 1 is a $20 \mathrm{fs}$ Gaussian pulse at $4 \mu \mathrm{m}$. Such short pulses at $4 \mu \mathrm{m}$ are not yet available, however it can be seen how the harmonic yield in this case drops dramatically. case 2 shows the results of the sub-cycle waveform described in [6], with 90\% ionization rate. Case 3 shows the results of the sub-cycle waveform described in [18], at a higher peak intensity and ionization rate, compared to the other cases. In case 4 , a synthesized waveform driven from the spectra shown Figure 2 with the amplitude ratio of 0.1:0:1 and the relative delay of $-6: 0: 5.4$ (fs) is considered. Case 5 describes the response of a $11.5 \mathrm{fs}$ Gaussian pulse centered at $2 \mu \mathrm{m}$. The two waveforms present four times higher yield compared to the other cases but an about two times higher yield can be achieved in case 6 which is driven by the optimized waveform calculated in this work.

Table 1. Comparison of the single dipole response of different waveforms interacting with He, for generation of isolated attosecond pulses at $1.7 \mathrm{keV}$ photon energies. The driver pulses in each case are as follows: Case 1: $20 \mathrm{fs}$, Gaussian pulses at $4 \mu \mathrm{m}$. Case 2: $1 \mathrm{fs}$ sub-cycle pulses centered at $540 \mathrm{~nm}$ discussed in [6]. Case 3 Sub-cycle pulse from the laser architecture discussed in [18] and shown in Figure 1. Case 4: The optimized synthesized pulses considering only the VIS and MIR channels of the synthesizer shown in Figure 1. This case shows the influence of the NIR channel. Case 5: Gaussian $11.5 \mathrm{fs}$ pulses at $2 \mu \mathrm{m}$. Case 6: The optimized waveform (see Figure $5 \mathrm{c}$ ) from the three channels of synthesizer shown in Figure 1. It can be seen that the optimized synthesized waveform has the highest yield with a seemingly low ionization rate.

\begin{tabular}{cccc}
\hline Case & Ionization Rate & Intensity $\left(\times \mathbf{1 0}^{\mathbf{1 4}}\right)$ & Yield (arb. Units) \\
\hline 1 & $0.01 \%$ & 3.5 & 0.0006 \\
2 & $99 \%$ & 52.8 & 1.05 \\
3 & $70 \%$ & 52.8 & 1.05 \\
4 & $16 \%$ & 13.9 & 41 \\
5 & $17.4 \%$ & 13.5 & 46 \\
6 & $24.5 \%$ & 15 & 78 \\
\hline
\end{tabular}

\section{Conclusions}

In conclusion, we demonstrated the prominent role of few-cycle pulse synthesis for optimizing the generation of isolated attosecond pulses at $\mathrm{keV}$ photon energies via HHG. We have shown that, in a sub-cycle pulse, the total electric field is confined to a sub-cycle period which results in an abrupt 
onset of ionization within a single half-cycle [16]. However, they are not the optimum shape of the waveform for $\mathrm{HHG}$ at $\mathrm{keV}$ photon energies. The increase in harmonic yield in our optimized waveform for generating isolated attosecond pulses at $1.7 \mathrm{keV}$ is due to the increased ionization within the main sub-cycle field and modification of the electron trajectories to have an earlier return time [32].

Our study shows that by moving the center of mass of the waveform's spectrum to longer wavelengths, the cutoff is increased. Using the optimized waveform for HHG suppresses the ionization probability. This is a major advantage for efficiently generating isolated attosecond pulses, as it allows for the most appropriate phase matching and energy-scaling schemes.

Here, the generation of attosecond pulses benefits from the minimum ionization prior the arrival of the main electric field's sub-cycle, which minimizes the density of free electrons and, hence, the distortion of the driving wave and its dephasing with the generated harmonic wave. As a result, the coherent build up of the harmonic emission over an extended propagation is maximized. In addition, the probability of ionization outside the central cycle is more than two orders of magnitude lower than that at the field maximum and hence is negligible.

Our study shows how the synthesis of multi-color broadband spectra enables fine tuning of the electron trajectories leading to the enhancement of the high harmonic yield and extension of the cutoff beyond what is achievable from the synthesis of semi-monochromatic fields at discrete frequencies [33-35].

Compared to the waveform in [6], the optimized waveform has a longer propagation length in the macroscopic scale due to the lower dispersion at a higher wavelength. This holds promise for compensation of the rapidly decreasing microscopic single-atom yield, by optimizing the dispersion of the free-electron plasma [28].

The generated sub-cycle pulses in Figure 2, with $1 \mathrm{fs}$ temporal duration at their FWHM, combined with the X-ray pulses which can be driven from the discussed optimized waveforms and from the same source, can serve as an ideal fronted for investigating spatiotemporal electron dynamics in solids.

Author Contributions: M.W. performed the simulations. M.H. developed the simulation code. H.F. designed and supervised the study. H.F and M.W. participated in discussions and wrote the paper.

Acknowledgments: H.F. acknowledges funding from Max Planck Society through Minerva fast-track scholarship.

Conflicts of Interest: The authors declare no conflict of interest.

\section{References}

1. Krausz, F.; Stockman, M.I. Attosecond metrology: from electron capture to future signal processing. Nat. Photonics 2014, 8, 205-213. doi:10.1038/nphoton.2014.28.

2. Krausz, F.; Ivanov, M. Attosecond physics. Rev. Mod. Phys. 2009, 81, 163-234. doi:10.1103/RevModPhys.81.163.

3. Wirth, A.; Hassan, M.T.; Grguras, I.; Gagnon, J.; Moulet, A.; Luu, T.T.; Pabst, S.; Santra, R.; Alahmed, Z.A.; Azzeer, A.M.; et al. Synthesized light transients. Science 2011, 334, 195-200. doi:10.1126/science.1210268.

4. Popmintchev, T.; Chen, M.C.; Popmintchev, D.; Arpin, P.; Brown, S.; Alisauskas, S.; Andriukaitis, G.; Balciunas, T.; Mücke, O.D.; Pugzlys, A.; et al. Bright coherent ultrahigh harmonics in the keV X-ray regime from mid-infrared femtosecond lasers. Science 2012, 336, 1287-1291. doi:10.1126/science.1218497.

5. Gordon, A.; Kärtner, F.X. Scaling of keV HHG photon yield with drive wavelength. Opt. Express 2005, 13, 2941-2947. doi:10.1364/OPEX.13.002941.

6. Moulet, A.; Tosa, V.; Goulielmakis, E. Coherent kiloelectronvolt X-rays generated by subcycle optical drivers: A feasibility study. Opt. Lett. 2014, 39, 6189-6192. doi:10.1364/OL.39.006189.

7. Geissler, M.; Tempea, G.; Brabec, T. Phase-matched high-order harmonic generation in the nonadiabatic limit. Phys. Rev. A 2000, 62, 033817. doi:10.1103/PhysRevA.62.033817.

8. Tosa, V.; Kim, K.T.; Nam, C.H. Macroscopic generation of attosecond-pulse trains in strongly ionized media. Phys. Rev. A 2009, 79, 043828. doi:10.1103/PhysRevA.79.043828.

9. Popmintchev, D.; Hernandez-Garcia, C.; Dollar, F.; Mancuso, C.; Perez-Hernandez, J.A.; Chen, M.C.; Hankla, A.; Gao, X.; Shim, B.; Gaeta, A.L.; et al. Ultraviolet surprise: Efficient soft X-ray high-harmonic generation in multiply ionized plasmas. Science 2015, 350, 1225-1231. doi:10.1126/science.aac9755. 
10. Takahashi, E.J.; Lan, P.; Mücke, O.D.; Nabekawa, Y.; Midorikawa, K. Attosecond nonlinear optics using gigawatt-scale isolated attosecond pulses. Nat. Commun. 2013, 4, 1-9. doi:10.1038/ncomms3691.

11. Jin, C.; Wang, G.; Le, A.T.; Lin, C.D. Route to optimal generation of soft X-ray high harmonics with synthesized two-color laser pulses. Sci. Rep. 2015, 4, 7067. doi:10.1038/srep07067.

12. Bandulet, H.C.; Comtois, D.; Bisson, E.; Fleischer, A.; Pépin, H.; Kieffer, J.C.; Corkum, P.B.; Villeneuve, D.M. Gating attosecond pulse train generation using multicolor laser fields. Phys. Rev. A 2010, 81, 013803. doi:10.1103/PhysRevA.81.013803.

13. Haessler, S.; Balčiunas, T.; Fan, G.; Andriukaitis, G.; Pugžlys, A.; Baltuška, A.; Witting, T.; Squibb, R.; Zaïr, A.; Tisch, J.W.G.; et al. Optimization of Quantum Trajectories Driven by Strong-Field Waveforms. Phys. Rev. X 2014, 4, 021028. doi:10.1103/PhysRevX.4.021028.

14. Kim, I.J.; Kim, C.M.; Kim, H.T.; Lee, G.H.; Lee, Y.S.; Park, J.Y.; Cho, D.J.; Nam, C.H. Highly Efficient High-Harmonic Generation in an Orthogonally Polarized Two-Color Laser Field. Phys. Rev. Lett. 2005, 94, 243901. doi:10.1103/PhysRevLett.94.243901.

15. Brizuela, F.; Heyl, C.M.; Rudawski, P.; Kroon, D.; Rading, L.; Dahlström, J.M.; Mauritsson, J.; Johnsson, P.; Arnold, C.L.; L'Huillier, A. Efficient high-order harmonic generation boosted by below-threshold harmonics. Sci. Rep. 2013, 3, 1410. doi:10.1038/srep01410.

16. Huang, S.W.; Cirmi, G.; Moses, J.; Hong, K.H.; Bhardwaj, S.; Birge, J.R.; Chen, L.J.; Li, E.; Eggleton, B.J.; Cerullo, G.; et al. High-energy pulse synthesis with sub-cycle waveform control for strong-field physics. Nat. Photonics 2011, 5, 475-479. doi:10.1038/nphoton.2011.140.

17. Fattahi, H. Sub-cycle light transients for attosecond, X-ray, four-dimensional imaging. Contemp. Phys. 2016, 57, 580-595. doi:10.1080/00107514.2016.1231870.

18. Fattahi, H.; Barros, H.G.; Gorjan, M.; Nubbemeyer, T.; Alsaif, B.; Teisset, C.Y.; Schultze, M.; Prinz, S.; Haefner, M.; Ueffing, M.; et al. Third-generation femtosecond technology. Optica 2014, 1, 45-63. doi:10.1364/OPTICA.1.000045.

19. Mucke, O.D.; Fang, S.; Cirmi, G.; Rossi, G.M.; Chia, S.H.; Ye, H.; Yang, Y.; Mainz, R.; Manzoni, C.; Farinello, P.; et al. Toward Waveform Nonlinear Optics Using Multimillijoule Sub-Cycle Waveform Synthesizers. IEEE J. Sel. Top. Quantum Electron. 2015, 21, 1-12. doi:10.1109/JSTQE.2015.2426653.

20. Fattahi, H.; Alismail, A.; Wang, H.; Brons, J.; Pronin, O.; Buberl, T.; Vámos, L.; Arisholm, G.; Azzeer, A.M.; Krausz, F. High-power, 1-ps, all-Yb:YAG thin-disk regenerative amplifier. Opt. Lett. 2016, 41, 1126-1129. doi:10.1364/OL.41.001126.

21. Fattahi, H.; Wang, H.; Alismail, A.; Arisholm, G.; Pervak, V.; Azzeer, A.M.; Krausz, F. Near-PHz-bandwidth, phase-stable continua generated from a Yb:YAG thin-disk amplifier. Opt. Express 2016, 24, 24337-24346. doi:10.1364/OE.24.024337.

22. Amotchkina, T.; Fattahi, H.; Pervak, Y.A.; Trubetskov, M.; Pervak, V. Broadband beamsplitter for high intensity laser applications in the infra-red spectral range. Opt. Express 2016, 24, 16752-16759. doi:10.1364/OE.24.016752.

23. Fattahi, H.; Wang, H.; Alismail, A.; Krausz, F. Towards high-power, multi-TW light transients. In Proceedings of the Conference on Lasers Electro-Optics, San Jose, CA, USA, 5-10 June 2016; OSA: Washington, DC, USA, 2016; p. SM1M.6. doi:10.1364/CLEO_SI.2016.SM1M.6.

24. Chipperfield, L.; Robinson, J.; Tisch, J.; Marangos, J. Ideal Waveform to Generate the Maximum Possible Electron Recollision Energy for Any Given Oscillation Period. Phys. Rev. Lett. 2009, 102, 0630031-0630034. doi:10.1103/PhysRevLett.102.063003.

25. Högner, M. Available online: https://gitlab.com/mhoegner/HHGmax (accessed on 2 May 2018).

26. Tong, X.M.; Lin, C.D. Empirical formula for static field ionization rates of atoms and molecules by lasers in the barrier-suppression regime. J. Phys. B 2005, 38, 2593-2600. doi:10.1088/0953-4075/38/15/001.

27. Lewenstein, M.; Balcou, P.; Ivanov, M.Y.; L'Huillier, A.; Corkum, P.B. Theory of high-harmonic generation by low-frequency laser fields. Phys. Rev. A 1994, 49, 2117-2132. doi:10.1103/PhysRevA.49.2117.

28. Popmintchev, T.; Chen, M.C.; Bahabad, A.; Gerrity, M.; Sidorenko, P.; Cohen, O.; Christov, I.P.; Murnane, M.M.; Kapteyn, H.C. Phase matching of high harmonic generation in the soft and hard X-ray regions of the spectrum. Proc. Natl. Acad. Sci. USA 2009, 106, 10516-10521. doi:10.1073/pnas.0903748106.

29. Hentschel, M.; Kienberger, R.; Spielmann, C.; Reider, G.A.; Milosevic, N.; Brabec, T.; Corkum, P.; Heinzmann, U.; Drescher, M.; Krausz, F. Attosecond metrology. Nature 2001, 414, 509-513. doi:10.1038/35107000. 
30. Keiber, S.; Sederberg, S.; Schwarz, A.; Trubetskov, M.; Pervak, V.; Krausz, F.; Karpowicz, N. Electro-optic sampling of near-infrared waveforms. Nat. Photonics 2016, 10, 159-162. doi:10.1038/nphoton.2015.269.

31. Jin, C.; Wang, G.; Wei, H.; Le, A.T.; Lin, C.D. Waveforms for optimal sub-keV high-order harmonics with synthesized two- or three-colour laser fields. Nat. Commun. 2014, 5, 4003. doi:10.1038/ncomms5003.

32. Siegel, T.; Torres, R.; Hoffmann, D.J.; Brugnera, L.; Procino, I.; Zaïr, A.; Underwood, J.G.; Springate, E.; Turcu, I.C.E.; Chipperfield, L.E.; et al. High harmonic emission from a superposition of multiple unrelated frequency fields. Opt. Express 2010, 18, 6853-6862. doi:10.1364/OE.18.006853.

33. Watanabe, S.; Kondo, K.; Nabekawa, Y.; Sagisaka, A.; Kobayashi, Y. Two-Color Phase Control in Tunneling Ionization and Harmonic Generation by a Strong Laser Field and Its Third Harmonic. Phys. Rev. Lett. 1994, 73, 2692-2695. doi:10.1103/PhysRevLett.73.2692.

34. Corkum, P. Plasma perspective on strong field multiphoton ionization. Phys. Rev. Lett. 1993, 71, $1994-1997$. doi:10.1103/PhysRevLett.71.1994.

35. Schafer, K.J.; Kulander, K.C. High Harmonic Generation from Ultrafast Pump Lasers. Phys. Rev. Lett. 1997, 78, 638-641. doi:10.1103/PhysRevLett.78.638.

(c) 2018 by the authors. Licensee MDPI, Basel, Switzerland. This article is an open access article distributed under the terms and conditions of the Creative Commons Attribution (CC BY) license (http://creativecommons.org/licenses/by/4.0/). 